\title{
The influence of olfactory context on Pavlovian conditioning and its expression in preweanling (16-day-old) and adult rats
}

\author{
NANCY A. LARIVIERE, WEI-JUNG CHEN, and NORMAN E. SPEAR \\ State University of New York, Binghamton, New York
}

\begin{abstract}
In a series of experiments, we tested the responses of preweanling (16 days postpartum) and adult rats to a specific olfactory context that was present during conditioning and testing of a brightness-location aversion. The first set of experiments established that (1) contrary to previously published effects with other distinctive contexts during conditioning and testing, a novel olfactory context impaired conditioning and its expression in preweanlings; (2) if the preweanlings were previously familiarized with the olfactory context, its presence impaired neither conditioning nor its expression; and (3) although conditioning in the absence of the olfactory context was equivalent for adults and preweanlings, the novel olfactory context had no effect on conditioning or its expression in adults. In the next set of experiments, we tested whether a conditioned aversion had been formed to the olfactory context. It was determined that although preweanlings acquired a substantial aversion to the olfactory context, whether novel or familiar, adults gave no indication of conditioning to the novel olfactory context. A final set of experiments established that preweanlings acquired an association between (or unitized) the CS+ and the context. These results add to previous indications that preweanlings may be especially sensitive to redundant or irrelevant features of the conditioning episode.
\end{abstract}

Ontogenetic change in stimulus selection during memory processing might include developmental differences in the processing of information about context. The possibility of ontogenetic differences in learning and remembering context is relevant to a number of issues. First, such differences would confirm that age-specific dispositions governing learning of an entire episode of conditioning may differ from those governing learning of a particular target within that episode. Such a circumstance might help explain ontogenetic differences in forgetting of a target memory that are observed despite ontogenetic equivalence in acquisition of the target ("infantile amnesia," reviewed by Campbell \& Spear, 1972; the possible relationship between infantile amnesia and stimulus selection has been discussed by Spear, 1979a, 1979b). Second, there are reasons to expect that incidental learning in general, of which contextual learning is a special case, is of vast importance to the developing animal, and there are suggestions that such learning is more likely and more efficacious in developing animals or humans than in adults (Smirnoff, 1973; Solheim, Hensler, \& Spear, 1980; Spear, 1984). Third, contextual influences on learning and memory constitute a significant area of study. Yet

This research was supported by a grant from the National Institute of Mental Health (1 RO1 MH35219) to Norman E. Spear. The authors would like to thank James $\mathbf{S}$. Miller for critically reading earlier drafts, David Kucharski for preliminary experiments, Teri Tanenhaus for secretarial assistance in preparing the manuscript, and Norman Richter for drawing the figures. Requests for reprints may be addressed to Norman E. Spear, Center for Developmental Psychology, State University of New York at Binghamton, Binghamton, NY 13901. despite substantial analytical advances in other respects (Bouton \& King, 1986; Bouton \& Swartzentruber, 1986; Gordon, 1983; Thomas \& McKelvie, 1982; Thomas, McKelvie, \& Mah, 1986; Thomas, McKelvie, Ranney, \& Moye, 1981), the probable dependence of such contextual influences on individual differences such as those associated with ontogeny has been largely ignored (for reviews, see Balsam \& Tomie, 1985; Spear, 1978, 1981).

Previous experiments have examined two ways in which learning about context might be expressed in behavior: the disruption in retention, which occurs when the context, but not the target, is changed between conditioning and testing, termed the contextual change effect; and the facilitation of conditioning due to the presence of the same distinctive context during conditioning and testing (relative to control groups conditioned and tested in a more conventional, less distinctive context), termed the distinctive context effect.

Two studies found the contextual change effect to be greater for preweanlings than for adults. Solheim et al. (1980) tested retention of instrumental learning, and Newman, Caza, and Spear (1982) tested retention of Pavlovian conditioning with regard to this effect. In both studies, it was especially apparent in preweanlings that retention of conditioning was more effective for the rats given the same olfactory context during conditioning and testing than for those given different contexts. The focus in the present study is on the former-rats given the same context during conditioning and testing.

We had reason to expect that among preweanlings with unchanged context, those given the more distinctive, novel 
olfactory context during both conditioning and testing would show the more effective retention. This would confirm the distinctive context effect, which has been observed frequently among preweanling rats (Concannon, Smith, Spear, \& Scobie, 1978; Richardson, Riccio, \& Axiotis, 1986; Richardson, Riccio, \& Jonke, 1983). Although it is unclear whether this context effect is relatively strong in infancy and decreases with age, this relationship was suggested by the results of Concannon et al. (1978).

Lariviere, Wipf, and Spear (1986) found, however, that when a novel olfactory context (the odor of lemon) was present during both conditioning and testing, expression of a brightness aversion (the target memory) was impaired relative to that observed when a less distinctive olfactory context was present during both conditioning and the retention test. To assess whether it was the novelty of the odor used as the context or the absolute properties of the odor that contributed to the disruption in brightness conditioning, a second experiment was conducted by Lariviere et al. to examine whether a familiar lemon contextual odor present during brightness conditioning would result in a similar impairment in the expression of conditioning. No impairment occurred, suggesting that olfactory context, even when consisting of an apparently strong odor, is not disruptive to either conditioning or the expression of an aversion when that odor is familiar to the animal. The absence of impairment with the familiarized odor probably was not due to simple cessation in the pup's response to that odor upon its familiarization. Following similar familiarization with an odor, preweanlings have shown continued responsiveness to it in terms of both an enhanced preference for that odor and enhanced discriminated escape learning in the presence of the odor relative to its absence (Wigal, Kucharski, \& Spear, 1984). Moreover, it has been established that an initially novel odor is still conditionable for animals of this age if given this same familiarization (Lariviere \& Spear, 1985).

The purpose of the present set of experiments was to further examine age-related differences in learning when a distinctive olfactory context is present during the conditioning episode. We also analyzed the possibility that the olfactory context of the conditioning episode enters into associations with an unconditioned stimulus (US; footshock) or a conditioned stimulus (CS; a visually distinctive location).

\section{GENERAL METHOD}

\section{Subjects}

The subjects were 16-day-old and adult (60-100-day-old) male and female Sprague-Dawley rats born and reared in the rat breeding colony at the State University of New York at Binghamton. All preweanling rats were housed with their parents and littermates in standard opaque maternity cages partially filled with pine shavings. All litters were culled to 8-10 pups within $24 \mathrm{~h}$ after the day of birth (Day 0). Adult subjects were weaned on Postnatal Day 21 and were individually housed in standard wire-mesh hanging cages until experimentation. Purina Lab Chow and tap water were available ad lib for all subjects. In addition, all animals were maintained on a 16-h light/8-h dark illumination cycle with light onset occurring at $0600 \mathrm{~h}$.

\section{Apparatus}

All conditioning and testing was conducted in a two-compartment aversive conditioning apparatus fitted with a stainless steel grid floor suitable for delivery of footshock ( $0.5 \mathrm{~mA}$ for $3 \mathrm{sec}$ via a Lafayette shock generator, Model A615C). Each compartment, one black and one white, was $13.9 \times 8.2 \times 8.8 \mathrm{~cm}$ for the preweanlings, and $25.85 \times 14.2 \times 13.9 \mathrm{~cm}$ for the adults. The conditioning apparatus had a door between the two compartments in order to confine the animal during conditioning. The door was removed during testing to allow the animal to locomote freely throughout the entire apparatus. Spatial odor-preference testing (Experiments 3A, 3B, $4,5 \mathrm{~A}$, and $6 \mathrm{~A}$ ) was conducted in a Plexiglas apparatus measuring $27.3 \times 10 \times 2 \mathrm{~cm}$ for the preweanlings, and $25.85 \times 14.2 \times$ $13.9 \mathrm{~cm}$ for the adults. Odors were spread on pieces of cotton placed beneath the standard wire-mesh floor on opposite sides of the apparatus. The odors employed in all of the experiments were lemon oil (Humco Laboratories, Texarkana, TX) or banana extract (Virginia Dare Artificial Ripe Banana, No. 112).

\section{Procedure}

Training and testing of the brightness aversion was conducted in the black/white conditioning apparatus. All experimental animals received two conditioning trials. A trial consisted of placing the animal in the black compartment of the apparatus (CS+) for $20 \mathrm{sec}$, with mild footshock $(0.5 \mathrm{~mA})$ administered during Seconds 8-10 and 18-20. This was immediately followed by confinement in the white compartment (CS-) for $20 \mathrm{sec}$, where no footshock was administered. The animals were placed in a holding cage partially filled with clean litter shavings for a 1-min intertrial interval (ITI). After a 30-min retention interval, testing consisted of placing the animal in the middle of the apparatus and allowing it to locomote freely between both sides of the apparatus. Time spent on the CS + (black) side of the apparatus during an 80-seconds preference test was recorded. The criterion for being on the black side of the apparatus was that the snout and two front paws were in that compartment.

\section{EXPERIMENT 1}

The purpose of the first experiment in this series was to compare, within a single experiment, the consequences of having a distinctive odor context (either novel or familiar) or no distinctive context present during both training and testing of a brightness discrimination. Basically, this experiment was an attempt to replicate the findings of Lariviere et al. (1986) within a single experiment, so that direct comparisons of the magnitude of brightness conditioning could be made between the groups.

\section{Method}

Subjects. Eighty-six 16-day-old rats were used as subjects in this experiment.

Apparatus. Black/white conditioning and testing was conducted in the apparatus described in the General Method section.

Design and Procedure. The design employed in this experiment was a $2 \times 3$ factorial. Rat pups were randomly assigned to one of six groups. The groups differed in whether paired $(P)$ or unpaired (UP) presentations of the CS+ (the black side of the apparatus) and footshock were given, and whether a novel odor (Nov), a familiar odor (Fam), or no odor (None) served as the context during both conditioning and testing. Specifically, None-None (P) animals were given pairings of the black side of the apparatus and shock (and of the white side with no shock) and tested as described 
in the General Method section. Fam-Fam (P) and Nov-Nov (P) animals were conditioned and tested in the same way, except that cotton scented with $1 \mathrm{cc}$ of a lemon odor was placed under both sides (CS + and CS - side) of the apparatus. Nov-Nov (P) animals were given no prior experience with the odor. In contrast, FamFam (P) animals were given prior experience with the lemon odor. The prior experience or familiarization phase of the experiment consisted of placing the rat pups in standard opaque maternity cages partially filled with pine shavings scented with $10 \mathrm{cc}$ of lemon odor, for a duration of $2 \mathrm{~h}$ on each of 5 consecutive days. This procedure has been shown to yield effective olfactory familiarization in preweanling rats (Wigal et al., 1984). The rat pups were 11 days old at the start of this exposure treatment.

None-None (UP) animals were administered footshock in a clear Plexiglas apparatus fitted on a grid floor, $1 \mathrm{~h}$ before exposure to the black and white compartments, with neither footshock nor odor present. The parameters for conditioning and testing were otherwise the same as those employed for the paired animals. Nov-Nov (UP) and Fam-Fam (UP) animals were given explicitly unpaired presentations in the same manner as None-None (UP) animals, but for these rats, either a novel odor (lemon) or a familiar odor (lemon) was present during both the conditioning and the testing phases of the experiment.

\section{Results and Discussion}

Mean time (in seconds) spent over the CS + during an 80 -sec black/white preference test is illustrated in Figure 1 . A $3 \times 2$ (context $\times$ condition) ANOVA performed on the data revealed a significant main effect of context $[F(2,90)=6.774, p<.002]$ and condition $\{F(1,90)=11.293, p<.001\}$, and a significant context $\times$ condition interaction $[F(2,90)=4.545, p<.013]$.

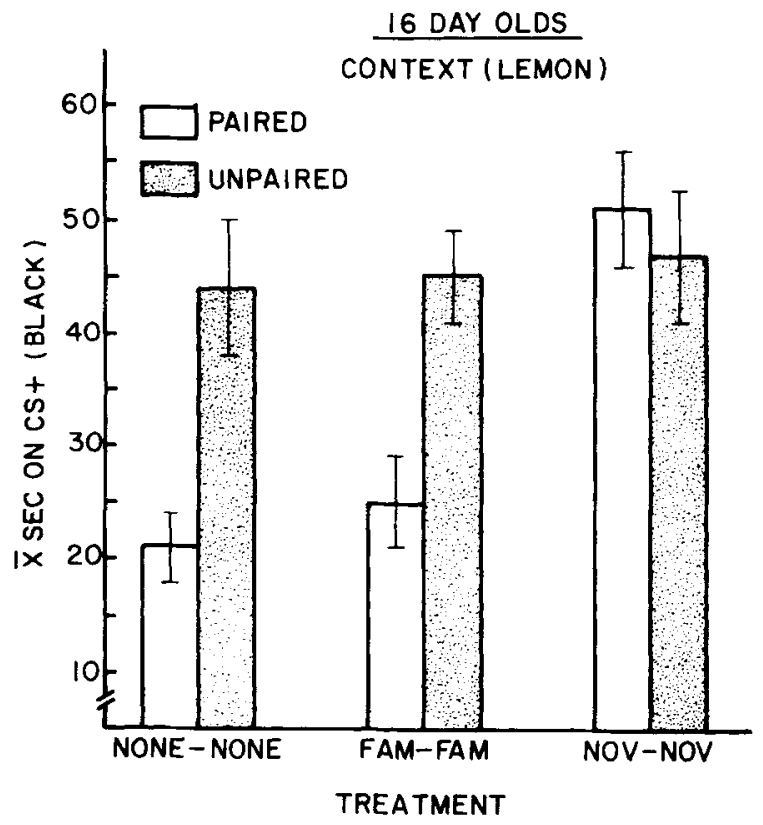

Figure 1. Mean ( $\pm S E$ ) time (in seconds) spent by 16-day-old rats over the CS + (the black side of the apparatus) during an 80-seconds black/white preference test in Experiment 1 as a function of conditioning treatment and whether a novel (Nov), familiar (Fam), or no odor context (None) was present during both training and testing. Lemon served as the odor context.
Post hoc comparisons (Fisher test, $p<.05$; Keppel, 1982) were conducted to analyze the locus of this interaction. While both None-None (P) and Fam-Fam (P) animals spent significantly less time on the $\mathrm{CS}+$ than did their respective unpaired groups, which indicates that a brightness aversion was learned, Nov-Nov (P) animals did not differ from Nov-Nov (UP) animals. In other words, conditioning and/or expression of the brightness aversion was disrupted-apparently precluded entirelywhen a novel odor was present during both conditioning and testing.

In addition, None-None $(\mathrm{P})$ and Fam-Fam $(\mathrm{P})$ animals spent significantly less time on the black side of the apparatus than did Nov-Nov (P) animals, whereas all three unpaired groups spent statistically the same amount of time on the black side. This result suggests that a novel or familiar odor present during stimulus presentations (the black and white side of the apparatus) and testing does not in itself change an animal's baseline preference for the black side of the apparatus.

This experiment, conducted primarily to replicate our previous findings with proper unpaired control groups, indicates that a novel olfactory context can impair the expression of conditioning in the preweanling rat. It is unclear to what extent this impairment is due to a disruption of memory storage or of memory retrieval. Previous evidence indicates that it may involve both stages (Lariviere et al., 1986). In that study, the presence of the novel odor at only the time of memory storage or only the time of retrieval tended to impair retention, but did so significantly less than when the novel odor was present during both stages of the experiment.

Experiment 1 established, for concurrently tested rats, that it was the novelty of the odor, and not its absolute properties, that disrupted the consequences of the conditioning procedures. (To avoid circumlocution, we shall henceforth refer to this as disruption of conditioning, with the understanding that the actual processes disrupted could be acquisition, retrieval, or some other aspect of the expression of an acquired memory.) The same odor that disrupted conditioning when it was novel during conditioning and testing had no effect on conditioning when it was familiar.

It remained uncertain, however, whether a novel contextual odor or a change in context between training and testing would affect conditioning of an aversion to a black compartment in adult rats as it does in preweanlings. In the following experiment, we tested this effect in adults.

\section{EXPERIMENT 2}

Previous research has indicated that in at least some circumstances, a change in olfactory context may be less disruptive to the expression of instrumental learning or Pavlovian conditioning in adult rats than it is in preweanlings (Newman et al., 1982; Solheim et al., 1980). These tests have indicated no apparent disruption of conditioning 
among adults when the olfactory context at conditioning differed from that at testing, despite considerable disruption among preweanlings in these circumstances (Lariviere et al., 1986).

The present experiment was designed to test whether a different effect of olfactory context-the facilitation or impairment observed by having a distinctive context present during conditioning and testing-would occur for adult rats. A previous study (Concannon et al., 1978) compared rats of different ages in terms of the distinctive context effect. Those researchers found that when present during both conditioning and testing, the internal context induced by pentobarbitol injection facilitated the expression of Pavlovian conditioning in 16-day-old rats but not in 23-day-old rats. Although involving facilitation rather than impairment of conditioning by a distinctive context during training and testing, the younger animals were affected more than the older animals. The older rats in the Concannon et al. study were too young to qualify as adults, however, and the context was a special type, a drug-induced state. The present experiment was designed to test the effect of olfactory context on conditioning in adults. Specifically, both the contextual change effect and the distinctive context effect were assessed in this experiment.

\section{Method}

Subjects. The subjects were 32 male and female adult rats between 60 and 100 days of age.

Apparatus. Brightness aversion conditioning and testing were conducted in the adult-sized (larger) apparatus described in the General Method section.

Procedure. The design for this experiment was a $2 \times 2$ (training $x$ testing) factorial in which the rats were randomly assigned to one of four groups ( $n=8$ each). The groups varied with regard to whether a novel lemon odor was present or not during training and testing. Specifically, Nov-Nov animals were both trained and tested in the presence of the lemon odor; Nov-None animals were trained in the presence of the lemon odor and tested without an odor present; None-Nov animals were trained without an odor context and tested in the presence of the lemon odor; and None-None animals were both trained and tested without lemon odor being present. Cotton scented with $1 \mathrm{cc}$ of the odor was placed under both sides (CS + and CS - side) of the apparatus for the animals trained and/or tested in the presence of an odor. The training and testing parameters employed for these groups was the same as that described in the General Method section. Unpaired control groups were not included as in Experiment 1; it has been demonstrated many times in our laboratory that the parameters employed in the present study result in substantial brightness aversions in adult rats that are comparable to those in 16-day-old rats (e.g., see Markiewicz, Kucharski, \& Spear, 1986). It also has been shown previously that 16-day-old and adult rats acquire odor aversions comparable in strength when a novel odor is employed in a basic classical conditioning paradigm (Markiewicz et al., 1986). Thus, we had reason to believe that the novel odor employed as the context in the present experiment would be as distinctive for the adult rats as it was for the pups.

\section{Results and Discussion}

Mean time (in seconds) spent over the CS + during the 80-sec test is illustrated in Figure 2. A $2 \times 2$ (training $\times$ testing) ANOVA conducted on the data revealed no sig-

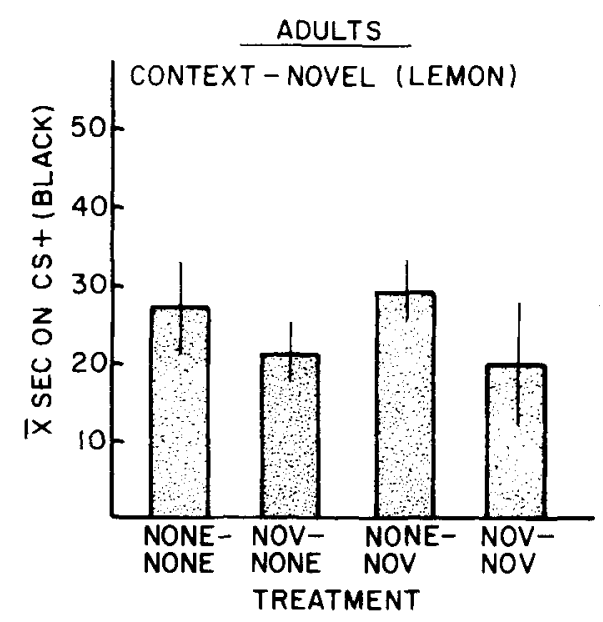

Figure 2. Mean ( $\pm S E$ ) time (in seconds) spent by adult rats over the CS+ (the black side of the apparatus) during an 80-seconds black/white preference test in Experiment 2 as a function of whether or not an odor context was present during training and/or testing. Lemon (a novel odor) served as the odor context.

nificant main effects or interactions. In other words, a novel lemon odor present during training and/or testing had no effect on expression of the brightness aversion in the adult rats. From what is known about unpaired control groups for brightness conditioning in adults (e.g., Markiewicz et al., 1986), there seems no doubt that all groups in the present experiment expressed aversions to the $\mathrm{CS}+$. There was no tendency whatsoever for a change in context between training and testing (Nov-None and None-Nov animals) to be disruptive relative to NoneNone and Nov-Nov animals. The means of the changed and unchanged groups were initially identical (see Figure 2).

Unlike preweanlings in the same circumstances, the adults gave no indication that either their acquisition or their expression of a conditioned aversion to the black compartment was impaired by a novel olfactory context or a change in that context between training and testing. In other words, neither the distinctive context effect nor the contextual change effect was observed.

\section{EXPERIMENT 3A}

It seems apparent, from Experiments 1 and 2 and previous results, that olfactory context can have substantial influence on the expression of conditioning in the preweanling rat in circumstances in which the adult shows little or no indication of such an influence. These two effectsthe context change effect and the distinctive context effect-may be interpreted as reflecting an age-related disposition of preweanlings to attend to and perhaps to learn redundant or irrelevant olfactory events. A more direct test of the learning of contextual events was applied in Experiments $3 \mathrm{~A}$ and $3 \mathrm{~B}$.

The general procedure was much like that in the previous experiments, with pairings of the black compartment and footshock (and the white compartment with no foot- 
shock) occurring in the context of a novel odor. But rather than testing conditioning to the black compartment, in the present experiments we tested the conditioning of an aversion to the odor that constituted the olfactory context. An analogous test of the association between context and the US has been conducted by Newman et al. (1982) and by Bouton and Bolles (1985). Experiment 3A included a test of such conditioning for 16-day-old rats and Experiment $3 \mathrm{~B}$ included the same test in the same circumstances for adult rats.

\section{Method}

Subjects. The subjects were 40 16-day-old rats $(n=10)$ from four litters. This experiment was conducted twice; each experiment employed 20 16-day-old rats $(n=10)$ from two litters.

Apparatus. Conditioning was conducted in the same black/white apparatus described previously. Odor-preference testing was conducted in a Plexiglas apparatus fitted on a wire-mesh floor. Cotton scented with 2 cc of Virginia Dare Artificial Ripe Banana 112 (the contextual odor) was placed at one end of the apparatus, and cotton scented with $1 \mathrm{cc}$ of lemon oil was located at the opposite end. Odor testing occurred in a dimly illuminated room, a lamp equipped with a General Electric $25-\mathrm{W}$ red bulb providing the only light.

Procedure. Rat pups were randomly assigned to one of two groups, either a group that received black/white conditioning in the presence of the banana odor (BW/O-P) or a group that received conditioning in the absence of the odor (BW/O-UP). A novel banana odor was employed in the present experiment rather than a lemon odor in order to increase the generality of these context effects. It was shown previously that a novel banana odor employed as the context disrupts brightness conditioning in the same manner as a lemon context odor (Lariviere et al., 1986), so the banana odor employed as the novel context in this experiment appeared to be an appropriate substitution for the novel lemon odor used previously. Conditioning of the brightness aversion was conducted as in the previous experiments.

For the BW/O-P rats, cotton scented with $1.5 \mathrm{cc}$ of banana odor was placed under both sides of the black/white box. After a 30-min retention interval, these animals were given a 3-min odorpreference test. For this test, cotton scented with $2 \mathrm{cc}$ of banana odor was placed at one end of the apparatus and cotton scented with $1 \mathrm{cc}$ of lemon oil was placed at the opposite end. Time spent over the banana odor (the odor that functioned as the context during conditioning) was recorded.

BW/O-UP animals were given black/white conditioning in the absence of any odor. Fifteen minutes later, these animals were exposed to the banana odor for $80 \mathrm{sec}$ (the length of the conditioning episode) in a clear Plexiglas box in which cotton scented with $1.5 \mathrm{cc}$ of the odor was placed under each compartment of the box. The same odor-preference test as was used for the BW/O-P rats was then given $15 \mathrm{~min}$ later. The small differences between these two groups in the interval separating odor exposure and testing is known to have a negligible effect on unconditioned odor preference (e.g., see Caza \& Spear, 1984). A significant difference between paired and unpaired animals would indicate the occurrence of a conditioned aversion to the odor context.

\section{Results}

Mean time (in seconds) spent over the conditioning context (banana odor) during the 3-min spatial odorpreference test is shown in Figure 3. A $2 \times 2$ (replication $\times$ condition) ANOVA was performed on the data to assess whether similar results were revealed in the two separate experiments. A significant main effect of condition $[F(1,36)=41.902, p<.001]$ was found without

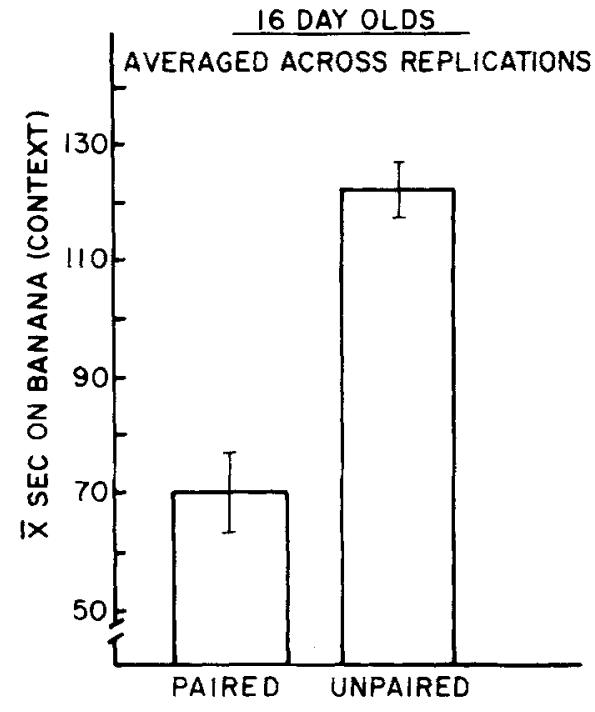

Figure 3. Mean ( $\pm S E$ ) time (in seconds) spent by 16-day-old rats over the odor context during a 3-min spatial odor-preference test in Experiment $3 A$ as a function of conditioning treatment. Banana (a novel odor) served as the odor context. These results are averaged across replications.

an interaction between replication and condition $[F(1,36)<1]$. This indicates that the BW/O-P animals, across the two experiments, spent significantly less time over the banana odor that had served as the context than did the BW/O-UP animals. In other words, it appears that the paired animals (black/white conditioning in the presence of an odor) acquired a conditioned aversion to the banana odor, whereas the unpaired animals (separate presentations of black/white conditioning and odor) did not.

\section{EXPERIMENT 3B}

\section{Method}

Subjects. The subjects were 22 male and female adult rats $(n=$ 11) between 60 and 100 days of age.

Apparatus. Conditioning and testing were conducted in the adultsized (larger) black/white apparatus and odor-preference chamber described in the General Method section.

Procedure. The procedure was identical to that employed in Experiment 3A.

\section{Results}

Mean time (in seconds) spent over the conditioning context (banana odor) during a 3-min spatial odor-preference test is illustrated in Figure 4. Mean $( \pm S E)$ time spent over the banana odor for the BW/O-P and BW/O-UP animals was the same $(92.73 \pm 8.12 \mathrm{sec}$, and $92.73 \pm 2.50 \mathrm{sec}$, respectively). These results indicate that the adult rats failed to acquire an aversion to the banana odor when the odor was present as a context during black/white conditioning.

\section{Discussion}

The basic observation of Experiment 3A was that if given pairings of a distinctive black compartment and foot- 


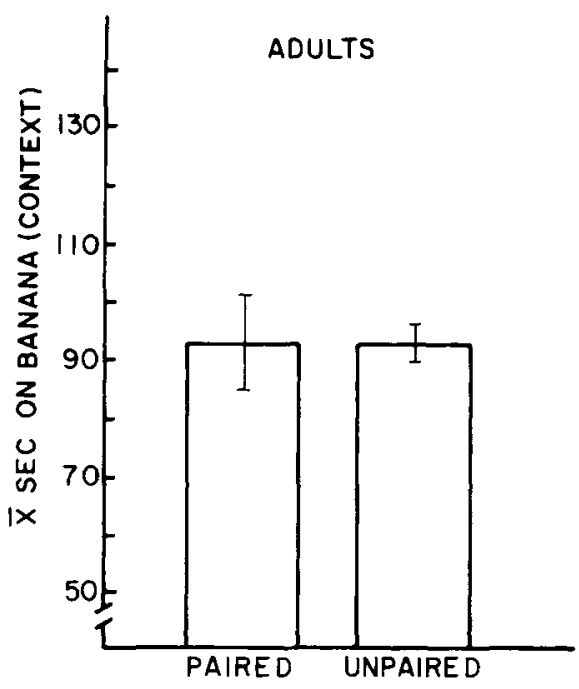

Figure 4. Mean ( $\pm S E$ ) time (in seconds) spent by adult rats over the odor context during a 3-min spatial odor-preference test in Experiment $3 \mathrm{~B}$ as a function of conditioning treatment. Banana (a novel odor) served as the odor context.

shock in a novel olfactory context, preweanling rats acquire an aversion to the olfactory context. Experiment 3B showed that for adults in the same circumstances, there was no measurable conditioning to the contextual odor. It should be clear that adult rats are not simply deficient in noticing or learning about novel odors such as those used in the present experiments. In several prior studies, adult and infant rats have shown equivalent or nearly equivalent basic conditioning and related effects from exposure to such odors (Caza \& Spear, 1984; Kucharski \& Spear, 1984; Markiewicz et al., 1986). There was some indication in the present study that the adult rats' preference for the banana odor differed from that of the 16-dayold rats: UP adult animals spent less time over the context odor than did the 16-day-old animals. This difference does not appear to be a problem for the interpretation of this experiment, since any differences in preference would influence paired and unpaired animals equally. Thus, the differences in conditioning of the context observed could not be attributed to simple differences in baseline preference for the banana odor. Nonetheless, differences in baseline preference may be a concern if unconditioned and conditioned preferences for odors are not simply additive, but instead interact when combined.

\section{EXPERIMENT 4}

The results so far indicate that the 16-day-old rat is quite sensitive to the influence of olfactory contexts during conditioning and testing of an aversion to a brightness-specific location. A novel olfactory context detracts significantly from the consequences of conditioning, whereas the same olfactory context when familiar does not. Direct conditioning of an olfactory context aversion, apparently due to context-US pairings, also occurs. Adults in the same circumstances do not show these effects, certainly not to the extent observed in 16-day-old pups.

Experiment 4 was designed specifically to test whether preweanlings would express an aversion to a familiar olfactory context. Experiment 1, as well as previous experiments, established that rats at this age can learn a brightness aversion in the presence of a familiar odor. Thus, if it is also possible to condition an aversion to the familiar context, it would provide a situation in which both a CS-US association and a context-US association would be evident.

\section{Method}

Subjects and Apparatus. The subjects were 37 16-day-old rats from four litters. The apparatus was the same as that used in Experiment 3A.

Procedure. The parameters for brightness conditioning were the same as those used in Experiment $3 \mathrm{~A}$, except that $1.5 \mathrm{cc}$ of a familiar, rather than a novel, odor served as the context in this experiment. The animals were familiarized to the odors in the same way as in Experiment 1. Rat pups were randomly assigned to one of four treatment groups, which varied with respect to the familiarization treatment (either lemon or banana odor) and whether brightness conditioning occurred in the presence (paired groups) or absence (unpaired groups) of one of the aforementioned odors. In other words, half of the animals familiarized on the banana odor received brightness conditioning in the presence of this odor, while half the animals received conditioning in the absence of the familiar odor. The animais familiarized on the lemon odor were divided into two groups in the same manner. Unpaired animals (those conditioned in the absence of the odor) received an 80-sec exposure to the odor used as the context in a clear Plexiglas chamber fitted on a grid floor, $15 \mathrm{~min}$ after the end of conditioning. Thirty minutes after brightness conditioning, paired and unpaired animals familiarized to the banana odor received a 3-min spatial odor-preference test between $2 \mathrm{cc}$ of banana odor and $1 \mathrm{cc}$ of the less preferred lemon odor. The animals familiarized to the lemon odor received an odorpreference test between $1 \mathrm{cc}$ of lemon and $0.75 \mathrm{cc}$ of orange, a less preferred odor (Humco Laboratories). (It is necessary for the novel alternative odor used in testing to be more aversive than the conditioned odor in order to eliminate the possibility that all animals will completely avoid the novel odor, which would lead to measurement problems.) Testing was conducted in the same way as in Experiments $3 \mathrm{~A}$ and $3 \mathrm{~B}$. Time spent over the odor employed as the context (either banana or lemon odor) was recorded.

\section{Results and Discussion}

Mean time (in seconds) spent over the banana or the lemon odor (context) during the 3-min odor-preference test is shown in Figure 5. A $2 \times 2$ (odor $\times$ condition) ANOVA conducted on the data revealed significant effects of odor $[F(1,33)=23.659, p<.001]$ and condition $[F(1,33)=16.796, p<.001]$, with no interaction between odor and condition. This indicates that both the banana- and the lemon-paired groups spent significantly less time on the contextual odor than did their respective unpaired groups. In other words, both paired groups of animals expressed an aversion to the familiar contextual odor. A main effect of odor was evident due to differences in baseline preference for these two odors in 16day-old animals.

This experiment and Experiment 3A demonstrate that preweanling rats are capable of forming a context-US as- 


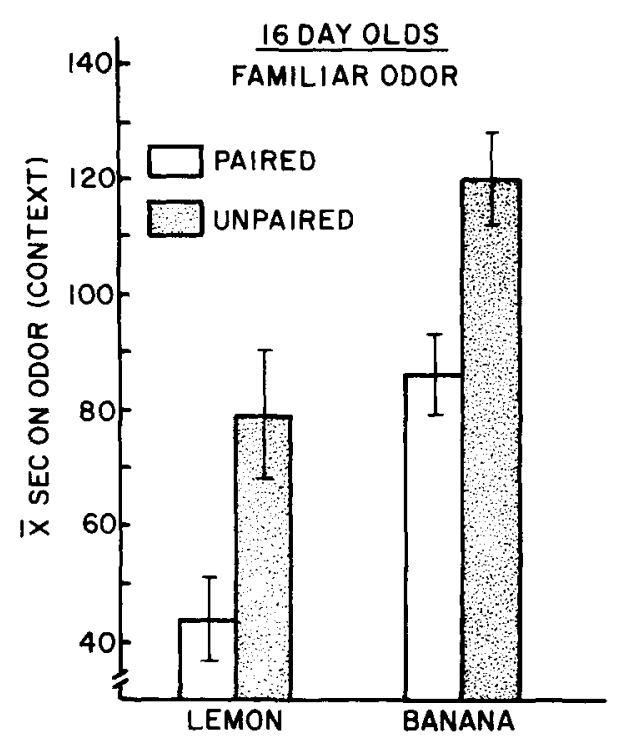

Figure 5. Mean ( $\pm S E$ ) time (in seconds) spent by 16-day-old rats over the odor context during a 3-min spatial odor-preference test in Experiment 4 as a function of conditioning treatment and odor used as the context. Lemon or banana (both familiar odors) served as the odor contexts.

sociation when the context consists of either a novel or a familiar odor. Since this fact was established in separate experiments, it was not possible to determine the relative strengths of these two odor aversions. It appears, from a cross-experiment comparison, that the novel and familiar odor aversions were comparable (although the animals in Experiment 4 had an increased baseline preference for the familiar odor), but this conclusion would be tentative at best.

These results provide further evidence to support the notion that infant rats may be more disposed than adults to attend to the irrelevant or redundant features of a conditioning episode (i.e., the context). In this respect, it is interesting that infant animals conditioned with a familiar odor employed as the context form not only an aversion to the context, but to the target episode (the CS+) as well. In other words, in this situation, the infant animals form both a context-US association and a CS-US association. What remains to be determined is whether an association or some other integrating device is formed between the $\mathrm{CS}+$ and the context.

\section{EXPERIMENT 5}

The considerable influence of context on the preweanlings could be a consequence of their "unitization" of the context and CS + . By unitization, we mean merely that two elements of the conditioning episode become treated as if they were functionally equivalent, such as inseparable or integral elements of a compound. A variety of effects are consistent with the idea that infant rats have a relatively strong disposition for such unitization, which diminishes with ontogeny (Spear, 1984; Spear, Kraemer,
Molina, \& Smoller, 1988; Spear \& Molina, 1987). One could also explain the present influences of context in terms of an "association" between the context and the CS. If these effects are in fact greater for preweanlings than for adults, such an explanation would be difficult to reconcile with the large number of experiments that indicate that the efficacy of associative conditioning increases with ontogeny (e.g., Rudy, Vogt, \& Hyson, 1984). Nevertheless, there is no strong reason to distinguish between association and unitization as the mechanism underlying the present effects of context. Our preference is to use the term unitization with the understanding that it could be read association.

If the context and the CS were unitized, we should expect that unpaired presentations of one of these would lead to extinction of both. Experiments 5 and 6 were intended to test this prediction.

Experiment 5 was conducted to establish circumstances in which unpaired presentations of either the familiar context (Experiment 5A) or the CS + (Experiment 5B) would lead to extinction of the context and $\mathrm{CS}+$ aversions, respectively.

\section{Experiment 5A}

\section{Method}

Subjects and Apparatus. The subjects were 25 16-day-old rats from three litters. Brightness conditioning and odor-preference testing were conducted in the same apparatuses as described previously.

Procedure. A $2 \times 2$ between-groups design was employed in this experiment. The rats were randomly assigned to one of four treatment groups that varied with regard to conditioning treatment (paired vs. unpaired) and context (odor) extinction treatment (none vs. $15 \mathrm{~min}$ ). Before the start of conditioning, all animals were familiarized to lemon odor as in Experiment 1. The parameters for conditioning of the brightness aversion, as well as testing of the aversion to the familiar lemon context, were the same as in Experiment 4 . The animals that received extinction treatment to the context (P-15 and UP-15) were exposed to the lemon odor for $15 \mathrm{~min}, 5 \mathrm{~min}$ after the end of conditioning. The animals were placed into individual Plexiglas compartments fitted on a grid floor under which was cotton scented with lemon odor. Ten minutes later, these animals were given an odor-preference test. Table 1 summarizes the treatments for these experiments and for Experiments $6 \mathrm{~A}$ and $6 \mathrm{~B}$.

\section{Results}

Mean time (in seconds) spent over the lemon odor (context) during a 3-min odor-preference test is shown in Figure 6. A $2 \times 2$ (condition $\times$ context extinction) ANOVA conducted on the data revealed a nonsignificant effect of condition $[F(1,21)=3.349, p<.08]$ and a condition $\times$ extinction interaction $[F(1,21)=3.349, p<$ $.08]$. (These $F$ values were coincidentally exactly the same.) Nonetheless, post hoc comparisons conducted on the data revealed that the P-None animals spent significantly less time on the lemon odor than did the UP-None animals, whereas the P-15 and UP-15 animals did not differ from each other. In other words, the animals that received nonreinforced exposure to the context between training and testing no longer expressed an aversion to the lemon odor that had served as the context. 
Table 1

Extinction Experiments

\section{Paired}

O Brightness conditioning

in a familiar context

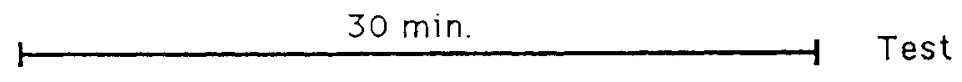

15 Brightness conditioning

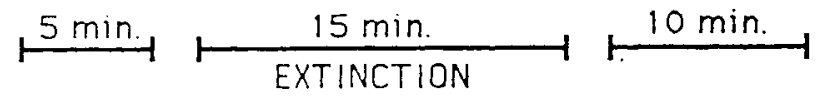

Test

\section{Unpaired}

$$
\begin{aligned}
& \text { O Footshock } \longrightarrow 30 \mathrm{~min} . \quad \begin{array}{c}
\text { B/W exposure in } \\
\text { a familiar context }
\end{array} \text { Test }
\end{aligned}
$$

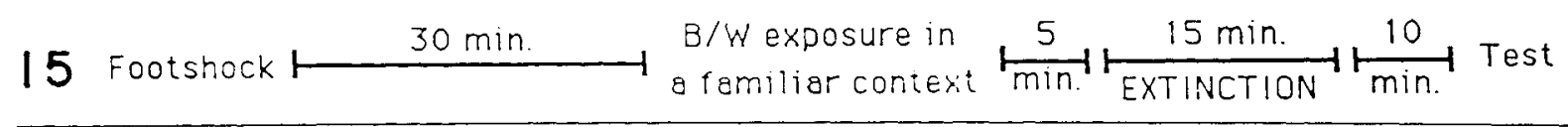

\section{Experiment 5B}

\section{Method}

Subjects and Apparatus. The subjects were 43 16-day-old rats representative of seven litters. The apparatus was the same as that employed in Experiment 5A.

Procedure. A $2 \times 2$ (condition $\times \mathrm{CS}+$ extinction treatment) was employed in this experiment. The rats were randomly assigned to one of four treatment groups that varied with regard to conditioning treatment (paired vs. unpaired) and $\mathrm{CS}+$ extinction treatment (none vs. $15 \mathrm{~min}$ ). All animals were familiarized to lemon odor in the same way as in Experiment 1 before the start of conditioning. The parameters for the conditioning of the brightness aversion were the same for the paired animals as in all previous experiments. Unpaired animals received a slightly different conditioning treatment. These animals received explicitly unpaired presentations of the conditioning episode and footshock. Footshock was presented to the unpaired animals in a clear Plexiglas chamber fitted on a grid floor 30 min prior to exposure to both the black and the white side of the apparatus in the presence of the odor. Since we were testing conditioning to the context in Experiment 5A, we did not need an explicitly unpaired control group (shock and black presentations were separate), but in Experiment 5B, this type of control was needed to gauge conditioning to the visual component $(\mathrm{CS}+)$. All subjects were given a black/white preference test $30 \mathrm{~min}$ after the end of conditioning. Testing was conducted in the same way as described in the General Method section. The animals that received a CS + extinction treatment (P-15 and UP-15) were placed in separate black compartments for $15 \mathrm{~min}, 5 \mathrm{~min}$ after the end of conditioning. Ten minutes later, they were given a black/white preference test.

\section{Results}

Mean time (in seconds) spent over the CS + (black side of the apparatus) during the 80 -sec preference test is shown in Figure 7. A $2 \times 2$ (condition $\times \mathrm{CS}+$ extinction treatment) ANOVA conducted on the data revealed significant effects of condition $[F(1,39)=9.102, p<$ $.004]$ and extinction $[F(1,39)=25.705, p<.001]$.
Although the interaction of these two variables did not achieve statistical significance, post hoc comparisons revealed that the P-None animals spent significantly less time on the CS + than did the UP-None animals, whereas the P-15 and UP-15 animals did not differ from each other. In other words, the animals that received nonreinforced exposure to the $\mathrm{CS}+$ between training and testing no longer expressed an aversion to the $\mathrm{CS}+$ (the black side

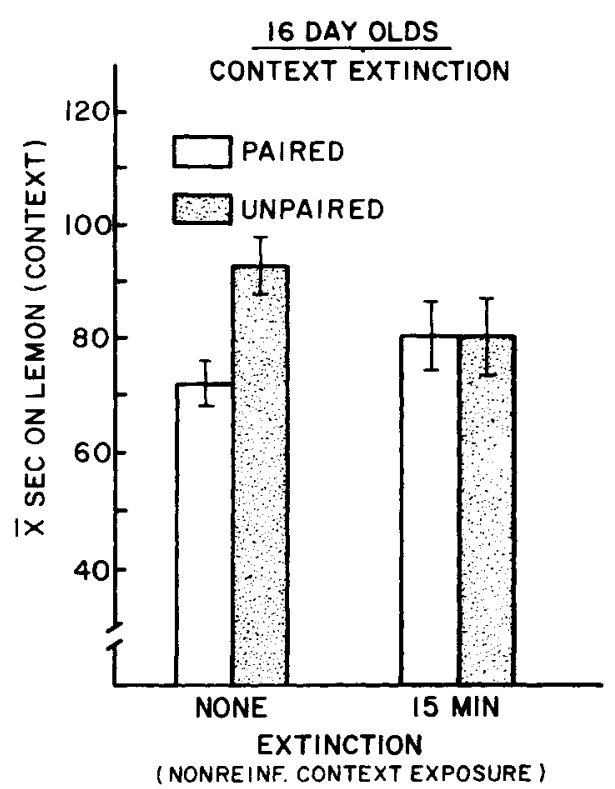

Figure 6. Mean ( $\pm S E$ ) time (in seconds) spent by 16-day-old rats over the odor context during a 3-min spatial odor-preference test in Experiment $5 \mathrm{~A}$ as a function of conditioning treatment and extinction treatment (nonreinforced context exposure). Lemon (a familiar odor) served as the odor context. 


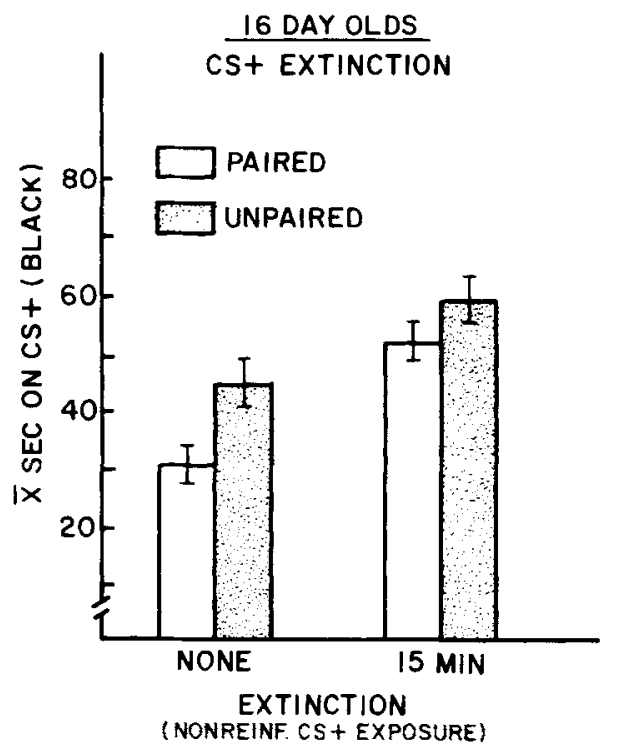

Figure 7. Mean ( $\pm S E$ ) time (in seconds) spent by 16-day-old rats over the CS + (the black side of the apparatus) during an 80-seconds black/white preference test in Experiment $5 B$ as a function of conditioning treatment and extinction treatment (nonreinforced CS+ exposure). Lemon (a familiar odor) served as the odor context.

of the apparatus). Post hoc comparisons also revealed a significant difference between the UP-0 and UP-15 animals, which is somewhat problematic. It is not clear why this difference occurred in our unpaired groups, but since we define conditioning as a difference between paired and unpaired animals, it does not change the conclusions drawn about the conditioning effect observed in this experiment.

\section{EXPERIMENT 6}

Experiments 5A and 5B showed that extinction of either the context aversion or the CS + aversion could be obtained with nonreinforced exposure to either the context or the $\mathrm{CS}+$, respectively. In Experiment $6 \mathrm{~A}$, we examined whether extinction of the CS + would also lead to extinction of the context, and in Experiment $6 \mathrm{~B}$ we examined whether extinction of the context would lead to extinction of the CS +. Either of these experiments would provide evidence for whether preweanlings do in fact unitize or form associations between the $\mathrm{CS}+$ and the context.

\section{Experiment 6A}

\section{Method}

Subjects and Apparatus. The subjects were 74 16-day-old rats from eight litters. This experiment was conducted twice; the first study employed 35 rats from four litters and the replication employed 39 rats from four litters. The apparatus for brightness conditioning and odor-preference testing was the same as that previously described.

Procedure. The design of this experiment was the same as that of Experiment 5B. All animals were familiarized to the lemon odor before the start of the experiment. Conditioning and testing were similar to that described previously, with the exception that all animals were given a 3 -min odor-preference test, rather than a black/white test, $30 \mathrm{~min}$ after conditioning. Half the animals in each of the paired and unpaired conditions received the extinction treatment-15 min of nonreinforced exposure to the CS + (black)and half did not (see Table 1). The odor-preference test, which tested the animals' aversion to the lemon context, was conducted as described in Experiment 4. Time spent over the lemon context was recorded.

\section{Results}

Mean time (in seconds) spent over the lemon odor during the 3-min spatial odor-preference test is illustrated in Figure 8. A $2 \times 2 \times 2$ (replication $\times$ condition $\times \mathrm{CS}+$ extinction treatment) ANOVA conducted on the data revealed significant main effects of replication $[F(1,66)=$ $8.242, p<.005]$ and condition $[F(1,66)=8.842$, $p<.004]$, and a significant condition $\times \mathrm{CS}+$ extinction treatment interaction $[F(1,66)=18.724, p<.001]$. The replication effect was due merely to differences in overall preference for the lemon odor, and there was no interaction between replication and any other factor. Data were therefore collapsed across the two separate studies and post hoc comparisons were performed to determine the locus of the interaction. These comparisons revealed that although the P-None animals spent significantly less time on the lemon odor than did the UP-None animals, indicating a lemon aversion, the P-15 animals did not differ from the UP-15 animals. This indicates that the $\mathrm{CS}+$ extinction treatment effectively eliminated the aversion to the context. Although post hoc comparisons (as in Experiment 5B) revealed a significant difference between the UP-0 and UP-15 animals, on the basis of our definition of conditioning (a difference between $P$ and UP

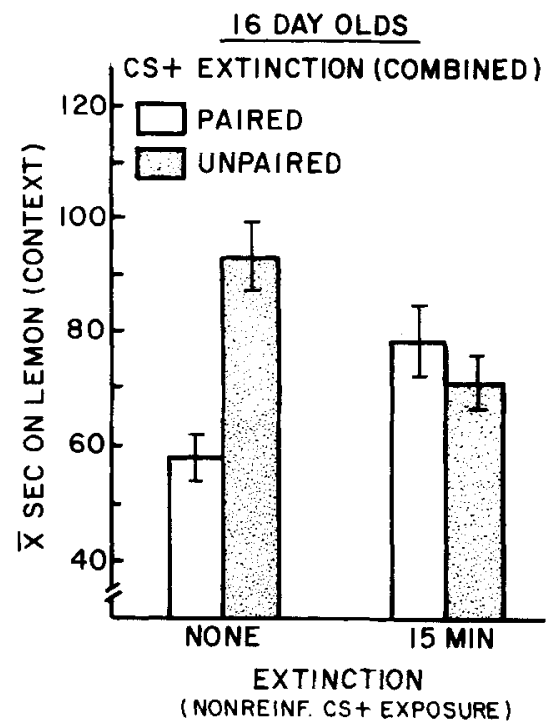

Figure 8. Mean ( $\pm S E$ ) time (in seconds) spent by 16-day-old rats over the odor context during a 3-min spatial odor-preference test in Experiment $6 \mathrm{~A}$ as a function of conditioning treatment and extinction treatment (nonreinforced CS + exposure). Lemon (a familiar odor) served as the odor context. These results are averaged across replications. 
groups), this finding does not change the conclusions drawn in this study. Basically, these data suggest the operation, among preweanlings, of a unitization or associative integrating process that served to link the $\mathrm{CS}+$ to the context of the conditioning episode.

\section{Experiment 6B}

\section{Method}

Subjects and Apparatus. The subjects were 46 16-day-old rats from six litters. Conditioning and testing apparatuses were the same as that described previously.

Procedure. The design of this experiment was the same as that employed in Experiment 5A. All animals were familiarized to the lemon odor prior to the start of conditioning. Conditioning and testing of the animals were the same as in Experiment 5A, with the exception that a black/white preference test was given to the animals after conditioning, rather than an odor-preference tets. Half the animals in each of the paired and unpaired conditions received the extinction treatment -15 min of nonreinforced exposure to the lemon context-and half did not (see Table 1). Time spent over the black side $(\mathrm{CS}+)$ of the apparatus was recorded during an $80-\mathrm{sec}$ preference test.

\section{Results and Discussion}

Mean time (in seconds) spent over the CS + (black side of the apparatus) during the 80 -sec preference test is shown in Figure 9. A $2 \times 2$ (condition $\times$ context extinction treatment) ANOVA performed on the data revealed a significant main effect of condition $[F(1,43)=9.266$, $p<.004]$, but with no interaction. In other words, both paired groups (P-None and $\mathrm{P}-15$ ) spent significantly less time on the $\mathrm{CS}+$ than did their respective unpaired groups. This indicates that the nonreinforced exposure to the context did not weaken the aversion to the CS+ (black side of the apparatus).

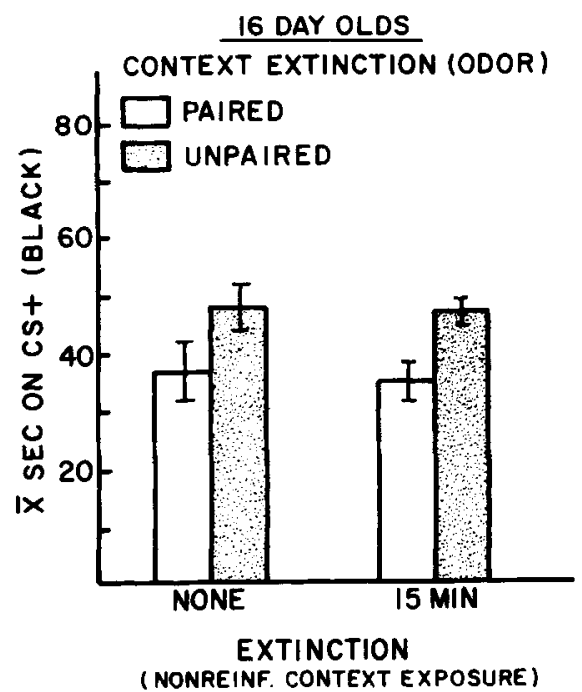

Figure 9. Mean ( $\pm S E$ ) time (in seconds) spent by 16-day-old rats over the CS + (the black side of the apparatus) during an 80-seconds black/white preference test in Experiment 6B as a function of conditioning treatment and extinction treatment (nonreinforced context exposure). Lemon (a familiar odor) served as the odor context.
Although this result may suggest that the context and the CS + are not in fact "unitized," alternative explanations are also possible. For instance, it is possible that the association between context and the $\mathrm{CS}+$ is unidirectional, from the CS + to the context but not vice versa, or that the present duration of nonreinforced exposure to the contextual odor was insufficient to extinguish the context-CS + association (although it was sufficient to extinguish the context-US association). A point relevant to still another possibility is that whereas the context was always present when the animal was exposed to the CS + , the reverse was not true. The CS + was present on only a fraction of the occasions on which the animal was exposed to the context; on other occasions in the presence of the context, the CS - was present or (during the ITI) neither the CS - nor the CS+ was present. Nonreinforced exposure to the context may therefore have led to equivalent extinction of the context-CS - and context-CS + associations, leaving their relative preference unaffected.

It is also possible that the context, although directly associated with the CS +, is not directly linked with the footshock, while the $\mathrm{CS}+$ is. Due to this direct association between the CS + and footshock, extinction of the CS + aversion would also lead to extinction of the context (Experiment $6 \mathrm{~A}$ ). Thus, the association between the $\mathrm{CS}+$ and the context may be bidirectional, but since the context was never directly linked with footshock in the absence of the $\mathrm{CS}+$, extinction of the context would not lead to extinction of the $\mathrm{CS}+$ aversion (Experiment 6B). Moreover, extinction of the context could eliminate the context aversion (Experiment $5 \mathrm{~A}$ ) by eliminating the link formed between the context and the $\mathrm{CS}+{ }^{1}$

Although the precise explanation is a matter for further research, it is clear from Experiment $6 \mathrm{~A}$ that nonreinforced exposure to the $\mathrm{CS}+$ reduced the aversion to the context, indicating some linkage between the two. This linkage might be an association between the CS + and the context (i.e., a within-compound association) as discussed above, or it might involve unitization of the context and many elements of the conditioning task together, including both the CS - and the CS + (i.e., the elements of the conditioning episode may be processed in a configural fashion).

\section{GENERAL DISCUSSION}

In the present experiments, we investigated the influence of olfactory context on the acquisition and retention of a conditioned aversion to a visually distinct location. The focus of these tests was on the preweanling, 16-day-old rat. Rats at this age had seemed particularly susceptible to the influence of context in these circumstances. This susceptibility might be expected in these circumstances because so much of the developing rat's behavior is guided by olfaction, and the 16-day-old rat has had access to vision (aside from detection of gross differences in brightness) for only a few days. Alternatively, preweanling rats 
might have a more general susceptibility to the influence of redundant or irrelevant stimuli (Spear, 1984). The present experiments could not decide this question, nor could they decide with certainty which particular contextual influences in the present study were age-dependent. What they did accomplish is an examination of the following features of contextual influence: the absolute influence of a particular olfactory context on conditioning, the association between context and the US, and the association between context and the CS+.

A major new finding of these experiments was that the presence of the same distinctive novel odor as context during conditioning and testing impaired expression of that conditioning in 16-day-old rats. The direction of the effect-impairment-is interesting because all previous effects of having the same distinctive context at conditioning and testing in preweanling animals of about this age have been facilitatory effects (Concannon et al., 1978; Pumo \& Spear, 1986; Richardson et al., 1986; Richardson et al., 1983). Explanations suggested previously for the facilitatory effects will therefore require revision in light of the opposite effects obtained in the present experiments.

It is not clear why the novel olfactory context impaired conditioning and/or its expression in the present circumstances. Perhaps the effect is merely analogous to overshadowing with a compound CS, so we need only say that the novel odor context overshadowed conditioning to the visual $\mathrm{CS}+$. The novel odor probably was more salient in some ways than the familiar context. Although preexposure to the contextual odor did not interfere completely, if at all, with its conditioning to the US, it may have contributed to the relative lack of overshadowing seen when a familiar context was employed. Since a direct comparison of the conditioned aversions to novel and familiar olfactory context was not conducted within a single experiment, it is difficult to determine the exact effect (if any) that odor preexposure had on this conditioning.

Yet, to attribute the impairment simply to overshadowing of the CS by the context, or more precisely, to the same processes responsible for overshadowing, seems somehow insufficient. For instance, the present experiments indicate that the impairment of conditioning by the novel olfactory context was more likely in infants than in adults and so the inference, by this explanation, would be that overshadowing was greater in infants than in adults. Opposed to this inference are several tests that have shown overshadowing by olfactory or gustatory events to be less likely in infants than in adults (e.g., Spear \& Kucharski, 1984a, 1984b; Kraemer, Lariviere, \& Spear, 1988). None of these tests of age-related differences in overshadowing have involved visual CSs, however, and a special interaction between olfactory events and visual CSs might occur differentially for infants and adults.

A second new result in the present experiments is the conditioned aversion to olfactory context established among infants given aversive conditioning in that context.
This is interesting because adults in the same circumstances gave no indication of acquiring an aversion to the context. It is unclear that the aversion in the infants was due to a direct association between the context and the US, but that seems a good possibility. This result brings to three the number of context-related phenomena that seem more apparent in infants than in adults: the context change effect (Solheim et al., 1980); the distinctive context effect, whether yielding facilitated or impaired expression of conditioning; and conditioning to context, possibly through a direct association between the context and the US.

A third new result was the finding that the 16-day-old rat tended to unitize, or form an association between, the $\mathrm{CS}+$ and the context. Nonreinforced exposure to the CS+ led to a decrease in the infant's conditioned aversion to the context. Adults were not tested in these circumstances; they might well have shown the same effect, although the minimal effect of olfactory context in our other tests would suggest not. It is not likely that adult rats would form an aversion to the familiar olfactory context if they show no evidence of forming an aversion to a novel olfactory context (Experiment 3B). It is possible that this influence, together with the other effects of context tested in the present experiments, collectively constitute a manifestation of the infant's hypothesized disposition to unitize separable events (Spear et al., 1988). The basic idea is that the infant may treat the events of the conditioning episode, such as the context, the CS, and the US, as functionally equivalent and essentially interchangeable. It remains to be determined whether this is a useful way to explain certain peculiarly infantile behaviors during conditioning.

The present results seem consistent with the general notion that preweanling animals are less likely than adults to be selective in their learning and are more likely to acquire memory attributes that are redundant to the particular conditioning task at hand (Spear, 1979a, 1984; Spear \& Molina, 1987). Some caution about the generality of the present effects is warranted because only one type of context was tested-olfactory. It is possible that younger animals have a special disposition to attend to and learn about redundant olfactory events. This is unclear and is, in any case, a very difficult proposition to test correctly. It is notable, however, that infants of the age tested in the present experiment probably are less proficient than adults at detecting olfactory events (Alberts, 1984). This makes it unlikely that the present effects are due to a greater impact of olfactory events in general among younger animals, and suggests that the generality of the present effects of context and their apparently age-related nature may extend beyond olfactory context.

\section{REFERENCES}

ALBERTS, J.R. (1984). Sensory-perceptual development in the Norway rat: A view toward comparative studies. In $\mathbf{R}$. Kail \& $\mathbf{N}$. E. Spear (Eds.), Comparative perspectives on the development of memory (pp. 65-102). Hillsdale, NJ: Erlbaum. 
Balsam, P. D., \& Tomie, A. (EDs.) (1985). Context and learning. Hillsdale, NJ: Erlbaum.

Bouton, M. E., \& Bolles, R. C. (1985). Contexts, event-memories, and extinction. In P. D. Balsam \& A. Tomie (Eds.), Context and learning (pp. 133-166). Hillsdale, NJ: Erlbaum.

Bouton, M. E., \& KING, D. A. (1986). Effect of context on performance to conditioned stimuli with mixed histories of reinforcement and nonreinforcement. Journal of Experimental Psychology: Animal Behavior Processes, 12, 4-15.

Bouton, M. E., \& Swartzentruber, D. (1986). Analysis of the associative and occasion-setting properties of contexts participating in a Pavlovian discrimination. Journal of Experimental Psychology: Animal Behavior Processes, 12, 333-350.

Campbell, B. A., \& Spear, N. E. (1972). Ontogeny of memory. Psychological Review, 79, 215-236.

CaZA, P. A., \& Spear, N. E. (1984). Short-term exposure to an odor increases its subsequent preference in preweanling rats: A descriptive profile of the phenomenon. Developmental Psychobiology, 17, $407-422$.

Concannon, J. T., Smith, G. J., Spear, N. E., \& Scobie, S. R. (1978). Drug cues, drug states, and infantile amnesia. In F. C. Colpaiert \& J. A. Rosencrans (Eds.), Stimulus properties of drugs: Ten years of progress. (pp. 353-396). Amsterdam: Elsevier/North-Holland.

GoRDon, W. C. (1983). The malleability of memory in animals. In R. L. Mellgren (Ed.), Animal cognition and behavior (pp. 399-426). New York: North-Holland.

KEPPEL, G. (1982). Design and analysis: A researcher's handbook (2nd ed.). Englewood Cliffs, NJ: Prentice-Hall.

Kraemer, P. J., Lariviere, N. A., \& Spear, N. E. (1988). Expression of a taste aversion conditioned with an odor-taste compound: Overshadowing is relatively weak in weanlings and decreases over a retention interval in adults. Animal Leaming \& Behavior, 16, 164-168.

KuChaRSK, D., \& SPEAR, N. E. (1984). Conditioning of an aversion to an odor paired with peripheral shock in the developing rat. Developmental Psychobiology, 17, 465-480.

LARIVIERE, N. A., \& SPEAR, N. E. (1985). Ontogenetic differences in transfer of conditioning between two different odors of equal familiarity. Dallas: International Society for Developmental Psychobiology.

Lariviere, N. A., Wipf, S., \& SPEAR, N. E. (1986, October). Ontogenetic differences in the effects of context on learning and retention. Paper presented at the meetings of the International Society for Developmental Psychobiology, Annapolis, MD.

MARKIEWICZ, B., Kucharski, D., \& SpEAR, N. E. (1986). Ontogenetic comparison of memory for Pavlovian conditioned aversions to temperature, vibration, odor or brightness. Developmental Psychobiology, 19, 139-154.

Newman, S., Caza, P. A., \& Spear, N. E. (1982, April). Ontogenetic differences in response to contextual stimuli. Paper presented at the meetings of the Eastern Psychological Association, Baltimore.

Pumo, J., \& SPEAR, N. E. (1986, October). The affective value of the context determines retention of Pavlovian conditioning in the 7-day old rat. Paper presented at the meetings of the International Society for Developmental Psychobiology, Annapolis, MD.

Richardson, R., Ruccio, D. C., \& Axiotis, R. (1986). Alleviation of infantile amnesia in rats by internal and external contextual cues. Developmental Psychobiology, 19, 453-462.

RichARDSON, R., Riccio, D. C., JoNKE, T. (1983). Alleviation of infantile amnesia in rats by means of a pharmacological contextual state. Developmental Psychobiology, 16, 511-518.

RudY, J. W., VOGT, M. B., \& HYSON, R. L. (1984). A developmental analysis of the rat's learned reactions to gustatory and auditory stimu- lation. In R. Kail \& N. E. Spear (Eds.), Comparative perspectives on the development of memory (pp. 181-208). Hillsdale, NJ: Erlbaum.

SMIRNoff, A. A. (1973). Problems of the psychology of memory. New York: Plenum.

Solhem, G. S., Hensler, J. G., \& SPEAR, N. E. (1980). Age-dependent contextual effects on short-term active avoidance retention in rats. Behavioral \& Neural Biology, 30, 250-259.

SPEAR, N. E. (1978). The processing of memories: Forgetting and retention. Hillsdale, NJ: Erlbaum.

SPEAR, N. E. (1979a). Experimental analysis of infantile amnesia. In J. F. Kihlstrom \& F. J. Evans (Eds.), Functional disorders of memory (pp. 75-101). Hillsdale, NJ: Erlbaum.

SPEAR, N. E. (1979b). Memory storage factors leading to infantile amnesia. In G. H. Bower (Ed.), The psychology of learning and motivation (Vol. 13, pp. 91-154). New York: Academic Press.

SPEAR, N. E. (1981). Extending the domain of memory retrieval. In N. E. Spear \& R. R. Miller (Eds.), Information processing in animals: Memory mechanisms (pp. 341-378). Hillsdale, NJ: Erlbaum.

SPEAR, N. E. (1984). Ecologically determined dispositions control the ontogeny of learning and memory. In R. Kail \& N. E. Spear (Eds.), Comparative perspectives on the development of memory (pp. 325358). Hillsdale, NJ: Erlbaum.

Spear, N. E., Kraemer, P. J., Molina, J. C., \& Smoller, D. E. (1988). Developmental change in learning and memory: Infantile disposition for "unitization." In J. Delacour (Ed.), Systems with learning and memory abilities (pp. 27-52). Amsterdam: Elsevier/NorthHolland.

SPEAR, N. E., \& KUChaRSKI, D. (1984a). Ontogenetic differences in the processing of multi-element stimuli: Potentiation and overshadowing. In H. Roitblat, T. Bever, \& H. Terrace (Eds.), Animal cognition (pp. 545-567). Hillsdale, NJ: Erlbaum.

SPEAR, N. E., KuCharSKI, D. (1984b). Ontogenetic differences in stimulus selection during conditioning. In R. Kail \& N. E. Spear (Eds.), Comparative perspectives on the development of memory (pp. 227-252). Hillsdale, NJ: Erlbaum.

SPEAR, N. E., Molina, J. C. (1987). The role of sensory modality in the ontogeny of stimulus selection. In N. Krasnegor, E. M. Blass, M. A. Hofer, \& W. P. Smotherman (Eds.), Perinatal development: A psychobiological perspective (pp. 85-110). Orlando, FL: Academic Press.

Thomas, D. R., \& MCKelvie, A. R. (1982). Retrieval of memory in the pigeon by context manipulations. Animal Learning \& Behavior, 10, $1-6$.

Thomas, D. R., McKelvie, A. R., \& MAH, W. (1986). The context as a conditional cue in operant discrimination reversal learning. Journal of Experimental Psychology: Animal Behavior Processes, 11, 317-330.

Thomas, D. R., McKelvie, A. R., Ranney, M., \& Moye, T. B. (1981). Interference in pigeons' long-term memory viewed as a retrieval problem. Animal Learning \& Behavior, 9, 581-586.

Wigal, T., KUCharSKI, D., \& SPEAR, N. E. (1984). Familiar contextual odors promote discrimination learning in preweanling but not in older rats. Developmental Psychobiology, 17, 555-570.

\section{NOTE}

1. The authors are grateful to Vincent M. LoLordo for this idea.

(Manuscript received January 18, 1989; revision accepted for publication August 6, 1989.) 